\title{
A third-order non-local problem with boundary integral condition for a parabolic equation
}

\author{
Bahloul Tarek*, Bouzit Mohammed \\ Department of Mathematics and Informatics, Oum El Bouaghi University, Algeria \\ *Corresponding author E-mail: tarek_bahloul@live.fr
}

Copyright (C)2015 Bahloul Tarek and Bouzit Mohammed. This is an open access article distributed under the Creative Commons Attribution License, which permits unrestricted use, distribution, and reproduction in any medium, provided the original work is properly cited.

\begin{abstract}
The present paper is devoted to a proof of the existence and uniqueness of a third-order non-local problem with boundary integral condition for a parabolic equation. The proof is based in two sided a priori estimates and the fact that the range of operator generalized by the considered problem is dense.
\end{abstract}

Keywords: Integral Boundary Condition, Energy Inequalities, nonlocal condition.

\section{Introduction}

In this paper we are concerned with the existence and uniqueness of a third-order non-local problem with boundary integral condition for a parabolic equation. We first consider the problem

$\frac{\partial u}{\partial t}-\frac{1}{x^{3}} \frac{\partial^{3}}{\partial x^{3}}\left(x\left(\frac{\partial^{2} u}{\partial x \partial t}\right)-\frac{1}{x^{3}} \frac{\partial^{2}}{\partial x^{2}}\left(x\left(\frac{\partial u}{\partial x}\right)\right)+\frac{1}{x^{3}} \frac{\partial^{2} u}{\partial x \partial t}=f(x, t)\right.$

$\frac{\partial^{i} u(0, t)}{\partial x^{i}}=0, \quad i=\overline{1,2}$

$\frac{\partial^{i} u(1, t)}{\partial x^{i}}=0, \quad i=\overline{1,3}$

$\frac{\partial u(0, t)}{\partial t}=\frac{\partial u(1, t)}{\partial t}=0$

$(x, t)$ in $(0, T) \times(0,1)$ with the initial datum

$l u=u(x, 0)=\varphi(x) \quad x \in(0,1)$

and the nonlocal boundary condition

$\int_{0}^{1} u(x, t) d x=0$. 
Throughout this paper we use the following abbreviation: $\varphi(x) \in L_{2}(0,1)$ is known function which satisfy the two compatibility conditions given in (1.2) and (1.4).

In recent years, boundary value problems for parabolic and hyperbolic equations with nonlocal conditions have been the subject of extensive study, see (Bouziani and Benouar[3], Denche [8], Yurchuk [13] and Al-kadhi [11]) and references therein.

Our method is as follows.First we establish two-sided a priori estimates. Then, we prove that the operator $L$ is a linear homeomorphism between the spaces $E$ and $F$.

We associate to problem (1.1), (1.2), (1.3) and (1.4) the operator $L=(\mathcal{L}, l)$, defined from $\mathrm{E}$ into $\mathrm{F}$, where $\mathrm{E}$ is the Banach space of functions $u \in L_{2}(\Omega)$, satisfying (1.2) and (1.4), with the finite norm

$$
\begin{gathered}
\|u\|_{E}^{2}=\int_{\Omega} x^{2}\left|\frac{\partial u}{\partial t}\right|^{2} d x d t+\int_{\Omega}\left|\frac{\partial^{2} u}{\partial x \partial t}\right|^{2} d x d t \\
+\int_{\Omega} \mid\left(\left.\frac{\partial^{2}}{\partial x^{2}}\left(x\left(\frac{\partial u}{\partial x}\right)\right)\right|^{2}+\mid\left(\left.\frac{\partial^{3}}{\partial x^{3}}\left(x\left(\frac{\partial^{2} u}{\partial x \partial t}\right)\right)\right|^{2} d x d t\right.\right. \\
+\sup _{0 \leq t \leq T} \int_{0}^{1}\left\{\left|\frac{\partial^{2}}{\partial x^{2}}\left(x\left(\frac{\partial u}{\partial x}\right)\right)\right|^{2}+x\left|\frac{\partial u}{\partial x}\right|^{2}+x^{2}|u|^{2}\right\} d x
\end{gathered}
$$

And $F$ is the Hilbert space of vector-valued functions $\mathcal{F}=(f, \varphi)$ obtained by completion of the space $L_{2}(\Omega) \times$ $W_{2}^{4}(0,1)$ with respect to the norm

$$
\begin{gathered}
\|\mathcal{F}\|_{F}^{2}=\|(f, \varphi)\|_{F}^{2}=\int_{\Omega} x^{3}|f|^{2} d x d t \\
+\int_{0}^{1}\left\{\left|\frac{\partial^{2}}{\partial x^{2}}\left(x\left(\frac{\partial \varphi}{\partial x}\right)\right)\right|^{2}+x\left|\frac{\partial \varphi}{\partial x}\right|^{2}+x^{2}|\varphi|^{2}\right\} d x
\end{gathered}
$$

Moreover,when solving this equation with nonlocal or classical boundary conditions by the energy integral method. This method is essentially based on the construction of suitable multiplicators for each specific given problem, which provides the a priori estimate from which it is possible to establish the solvability of the posed problem.

\section{Two-sided a priori estimates}

For any function $u \in E$, there is the a priori estimate

$$
\|L u\|_{F} \leq c\|u\|_{E}
$$

where $c$ is a constant which may depend on $T$ but does not depend on $u$.

Proof Using (1.1) and the initial conditions (1.2), we obtain

$$
\begin{gathered}
\int_{\Omega} x^{3}|\mathcal{L} u|^{2} d x d t \\
\leq \int_{\Omega} x^{2}\left|\frac{\partial u}{\partial t}\right|^{2} d x d t+\int_{\Omega} \mid\left(\left.\frac{\partial^{2}}{\partial x^{2}}\left(x^{2}\left(\frac{\partial u}{\partial x}\right)\right)\right|^{2} d x d t\right. \\
+\int_{\Omega} \mid\left(\left.\frac{\partial^{3}}{\partial x^{3}}\left(x\left(\frac{\partial^{2} u}{\partial x \partial t}\right)\right)\right|^{2} d x d t\right. \\
+\int_{\Omega}\left|\frac{\partial^{2} u}{\partial x \partial t}\right|^{2} d x d t \\
\int_{0}^{1}\left|\frac{\partial^{2}}{\partial x^{2}}\left(x\left(\frac{\partial \varphi}{\partial x}\right)\right)\right|^{2} d x \leq \sup _{0 \leq t \leq T} \int_{0}^{1}\left|\frac{\partial^{2}}{\partial x^{2}}\left(x\left(\frac{\partial u}{\partial x}\right)\right)\right|^{2} d x \\
\int_{0}^{1}\left(x\left|\frac{\partial \varphi}{\partial x}\right|^{2}+x^{2}|\varphi|^{2}\right) d x \leq \sup _{0 \leq t \leq T} \int_{0}^{1}\left(x\left|\frac{\partial u}{\partial x}\right|^{2}+x^{2}|u|^{2}\right) d x
\end{gathered}
$$


Combining the inequalities (2.2), (2.3), and (2.4) we obtain (2.1) for $u \in E$.

For any function $u \in E$, there is the a priori estimate

$\|u\|_{E} \leq C\|L u\|_{F}$

with the constant

$C=\frac{64 \quad \exp (-c T)}{\min (48 ;(3 c-51))}$

and $c$ is such that

$c \geq 17$.

To see this, the following lemmas are essential.

Lemma 2.1 For $u \in E$ satsfying the first condition in (1.2)

$$
\begin{gathered}
\frac{1}{4} \int_{0}^{1} \exp (-c \tau)\left|\frac{\partial^{2}}{\partial x^{2}}\left(x\left(\frac{\partial u(x, \tau)}{\partial x}\right)\right)\right|^{2} d x \\
+\frac{(c-1)}{4} \int_{0}^{\tau} \int_{0}^{1} \exp (-c t)\left|\frac{\partial^{2}}{\partial x^{2}}\left(x\left(\frac{\partial u}{\partial x}\right)\right)\right|^{2} d x d t \\
\leq \frac{1}{4} \int_{0}^{1}\left|\frac{\partial^{2}}{\partial x^{2}}\left(x\left(\frac{\partial \varphi}{\partial x}\right)\right)\right|^{2} d x+\frac{1}{4} \int_{0}^{\tau} \int_{0}^{1} \exp (-c t)\left|\frac{\partial^{2}}{\partial x^{2}}\left(x\left(\frac{\partial^{2} u}{\partial x \partial t}\right)\right)\right|^{2} d x d t
\end{gathered}
$$

Proof Starting from

$\frac{1}{2} \int_{0}^{\tau} \int_{0}^{1} \exp (-c t) \frac{\partial^{2}}{\partial x^{2}}\left(x\left(\frac{\partial u}{\partial x}\right)\right) \frac{\partial^{2}}{\partial x^{2}}\left(x\left(\frac{\partial^{2} \bar{u}}{\partial x \partial t}\right)\right) d x d t$

Then integrating by parts and using elementary inequalities, we obtain (2.8)

Lemma 2.2 For $u \in E$ satsfying the first condition in (1.2)

$\frac{1}{4} \int_{0}^{\tau} \int_{0}^{1} \exp (-c t) \mid \frac{\partial^{2}}{\partial x^{2}}\left(\left.x\left(\frac{\partial^{2} u}{\partial x \partial t}\right)\right|^{2} d x d t \leq \int_{0}^{\tau} \int_{0}^{1} \exp (-c t)\left|\frac{\partial^{3}}{\partial x^{3}}\left(x\left(\frac{\partial^{2} u}{\partial x \partial t}\right)\right)\right|^{2} d x d t\right.$.

Proof Starting from

$\int_{0}^{\tau} \int_{0}^{1} \exp (-c t)(1-x) \frac{\partial^{3}}{\partial x^{3}}\left(x\left(\frac{\partial^{2} u}{\partial x \partial t}\right)\right) \frac{\partial^{2}}{\partial x^{2}}\left(x\left(\frac{\partial^{2} \bar{u}}{\partial x \partial t}\right)\right) d x d t$

Then integrating by parts and using elementary inequalities, we obtain (2.9)

Lemma 2.3 For $u \in E$ satsfying the first condition in (1.2)

$$
\begin{gathered}
\frac{1}{2} \int_{0}^{1} x^{2} \exp (-c \tau)|u(x, \tau)|^{2} d x+\frac{(c-1)}{2} \int_{0}^{\tau} \int_{0}^{1} \exp (-c t) x^{2}|u|^{2} d x d t \\
\leq \frac{1}{2} \int_{0}^{\tau} \int_{0}^{1} \exp (-c t) x^{2}\left|\frac{\partial u}{\partial t}\right|^{2} d x d t+\frac{1}{2} \int_{0}^{1} x^{2}|\varphi|^{2} d x
\end{gathered}
$$

With $c \geq 19$.

Proof Integrating by parts the expression

$\int_{0}^{\tau} \int_{0}^{1} x^{2} \exp (-c t) u \frac{\partial \bar{u}}{\partial t} d x d t$

And using elementary inequalities yield (2.9). 
Proof Theorem 2.2

Let

$\mathcal{M} u=x^{3} \mathcal{J}_{\frac{\partial u}{\partial t}}$

and

$\mathcal{J}_{u}=\int_{x}^{1} u(\xi, t) d \xi$

We consider the quadratic form

$\operatorname{Re} \int_{0}^{\tau} \int_{0}^{1} \exp (-c t) \mathcal{L} u \overline{\mathcal{M} u}$

which is obtained by multiplying (1.1) by $\exp (-c t) \overline{\mathcal{M} u}$

Hence we have also

$$
\begin{gathered}
\int_{0}^{1} \int_{0}^{\tau} \exp (-c t) \mathcal{L} u \mathcal{M} \bar{u} d x d t=\int_{0}^{1} \int_{0}^{\tau} \exp (-c t)\left(\frac{\partial u}{\partial t}-\frac{1}{x^{3}}\left(\frac{\partial^{3}}{\partial x^{3}}\left(x\left(\frac{\partial^{2} u}{\partial x \partial t}\right)\right)\right.\right. \\
-\frac{1}{x^{3}}\left(\frac{\partial^{2}}{\partial x^{2}}\left(x\left(\frac{\partial u}{\partial x}\right)\right)+\frac{1}{x^{3}} \frac{\partial^{2} u}{\partial x \partial t}\right)\left(x^{3} \mathcal{J}_{\frac{\partial \bar{u}}{\partial t}}\right) d x d t
\end{gathered}
$$

Integrating by parts (2.13) with the use of boundary conditions (1.2),(1.3) and (1.4), we obtain

$$
\begin{gathered}
\int_{0}^{\tau} \int_{0}^{1} \exp (-c t) \mathcal{L} u \mathcal{M} \bar{u} d x d t=\int_{0}^{\tau} \int_{0}^{1} \exp (-c t) \frac{\partial u}{\partial t} x^{3} \mathcal{J}_{\frac{\partial \bar{u}}{\partial t}} d x d t \\
-\int_{0}^{\tau} \int_{0}^{1} \exp (-c t) \frac{1}{x^{3}}\left(\frac{\partial^{2}}{\partial x^{2}}\left(x\left(\frac{\partial u}{\partial x}\right)\right) x^{3} \mathcal{J}_{\frac{\partial \bar{u}}{\partial t}} d x d t\right. \\
-\int_{0}^{\tau} \int_{0}^{1} \exp (-c t) \frac{1}{x^{3}} \frac{\partial^{3}}{\partial x^{3}}\left(x\left(\frac{\partial^{2} u}{\partial x \partial t}\right)\right) x^{3} \mathcal{J}_{\frac{\partial \bar{u}}{\partial t}} d x d t \\
+\int_{0}^{\tau} \int_{0}^{1} \exp (-c t) \frac{1}{x^{3}} \frac{\partial^{2} u}{\partial x \partial t} x^{3} \mathcal{J}_{\frac{\partial \bar{u}}{\partial t}} d x d t
\end{gathered}
$$

Now, from (2.14) we have

$\operatorname{Re} \int_{0}^{\tau} \int_{0}^{1} \exp (-c t) \frac{\partial u}{\partial t} x^{3} \mathcal{J}_{\frac{\partial \bar{u}}{\partial t}} d x d t=\frac{3}{2} \int_{0}^{\tau} \int_{0}^{1} \exp (-c t) x^{2}\left|\mathcal{J}_{\frac{\partial u}{\partial t}}\right|^{2} d x d t$

Again, Integrating by report to $\mathrm{x}$, and taking into account of the boundary conditions, we obtain

$$
\begin{gathered}
-\operatorname{Re} \int_{0}^{\tau} \int_{0}^{1} \exp (-c t) \frac{\partial^{2}}{\partial x^{2}}\left(x\left(\frac{\partial u}{\partial x}\right)\right) \mathcal{J}_{\frac{\partial \bar{u}}{\partial t}} d x d t= \\
\frac{1}{2} \int_{0}^{1} \exp (-c \tau) x\left|\frac{\partial u(x, \tau)}{\partial x}\right|^{2} d x-\frac{1}{2} \int_{0}^{1} x\left|\frac{\partial \varphi}{\partial x}\right|^{2} d x \\
+\frac{c}{2} \int_{0}^{\tau} \int_{0}^{1} \exp (-c t) x\left|\frac{\partial u}{\partial x}\right|^{2} d x d t
\end{gathered}
$$

Similarly we can handle other terms to obtain

$$
\begin{gathered}
-R e \int_{0}^{\tau} \int_{0}^{1} \exp (-c t) \frac{1}{x^{3}}\left(\frac{\partial^{3}}{\partial x^{3}}\left(x\left(\frac{\partial^{2} u}{\partial x \partial t}\right)\right) x^{3} \mathcal{J}_{\frac{\partial \bar{u}}{\partial t}} d x d t=\right. \\
\int_{0}^{\tau} \int_{0}^{1} \exp (-c t)\left|\frac{\partial^{2} u}{\partial x \partial t}\right|^{2} d x d t
\end{gathered}
$$

The most delicate term in the right-hand side of (2.15) is the third one, which is treated as follows.

$$
\int_{0}^{1} \int_{0}^{\tau} \exp (-c t) \frac{\partial^{2} u}{\partial x \partial t} \mathcal{J}_{\frac{\partial u}{\partial t}} d x d t=\int_{0}^{1} \int_{0}^{\tau} \exp (-c t)\left|\frac{\partial u}{\partial t}\right|^{2} d x d t
$$


By using the proprieties of the modules and of the $\varepsilon$-inequality, we obtain

$$
\begin{gathered}
\operatorname{Re} \int_{0}^{\tau} \int_{0}^{1} \exp (-c t) \mathcal{L} u \mathcal{M} \bar{u} d x d t \leq\left|\int_{0}^{\tau} \int_{0}^{1} \exp (-c t) \mathcal{L} u \mathcal{M} \bar{u} d x d t\right| \\
\leq \int_{0}^{\tau} \int_{0}^{1}|\exp (-c t) \mathcal{L} u \mathcal{M} \bar{u}| d x d t \\
\leq \int_{0}^{\tau} \int_{0}^{1} \exp (-c t) x^{3}|\mathcal{L} u|\left|\mathcal{J}_{\frac{\partial \bar{u}}{\partial t}}\right| d x d t \\
\leq \frac{1}{6} \int_{0}^{\tau} \int_{0}^{1} \exp (-c t) x^{3}|\mathcal{L} u|^{2} d x d t+\frac{3}{2} \int_{0}^{\tau} \int_{0}^{1} \exp (-c t) x^{2}\left|\mathcal{J}_{\frac{\partial \bar{u}}{\partial t}}\right|^{2} d x d t
\end{gathered}
$$

From where, and since $(x \leq 1)$ we obtain

$$
\begin{gathered}
\frac{1}{6} \int_{0}^{\tau} \int_{0}^{1} x^{3}|\mathcal{L} u|^{2} d x d t+\frac{1}{2} \int_{0}^{1} x\left|\frac{\partial \varphi}{\partial x}\right|^{2} d x \geq \int_{0}^{\tau} \int_{0}^{1} \exp (-c t)\left|\frac{\partial^{2} u}{\partial x \partial t}\right|^{2} d x d t \\
+\frac{1}{2} \int_{0}^{1} \exp (-c \tau) x\left|\frac{\partial u(x, \tau)}{\partial x}\right|^{2} d x+\frac{c}{2} \int_{0}^{\tau} \int_{0}^{1} \exp (-c t) x\left|\frac{\partial u}{\partial x}\right|^{2} d x d t \\
+\int_{0}^{\tau} \int_{0}^{1} \exp (-c t) x^{2}\left|\frac{\partial u}{\partial t}\right|^{2} d x d t
\end{gathered}
$$

From the equation (1.1) we have

$$
\begin{gathered}
\frac{1}{2} \int_{0}^{\tau} \int_{0}^{1} \exp (-c t)\left|\frac{\partial^{3}}{\partial x^{3}}\left(x\left(\frac{\partial^{2} u}{\partial x \partial t}\right)\right)\right|^{2} d x d t \\
-\int_{0}^{\tau} \int_{0}^{1} \exp (-c t)\left|\frac{\partial^{2}}{\partial x^{2}}\left(x\left(\frac{\partial u}{\partial x}\right)\right)\right|^{2} d x d t \leq \\
\int_{0}^{\tau} \int_{0}^{1} \exp (-c t)\left|\frac{\partial^{2} u}{\partial x \partial t}\right|^{2} d x d t+\int_{0}^{\tau} \int_{0}^{1} x^{3}|\mathcal{L} u|^{2} d x d t \\
+\int_{0}^{\tau} \int_{0}^{1} \exp (-c t) x^{2}\left|\frac{\partial u}{\partial t}\right|^{2} d x d t
\end{gathered}
$$

Combining inequalitie (2.22), and Lemma 2.3 we get

$$
\begin{gathered}
\frac{1}{4} \int_{0}^{\tau} \int_{0}^{1} \exp (-c t)\left|\frac{\partial^{3}}{\partial x^{3}}\left(x\left(\frac{\partial^{2} u}{\partial x \partial t}\right)\right)\right|^{2} d x d t \\
+\frac{(c-17)}{32} \int_{0}^{\tau} \int_{0}^{1} \exp (-c t)\left|\frac{\partial^{2}}{\partial x^{2}}\left(x\left(\frac{\partial u}{\partial x}\right)\right)\right|^{2} d x d t \\
+\frac{1}{32} \int_{0}^{1} \exp (-c \tau) \mid \frac{\partial^{2}}{\partial x^{2}}\left(\left.x\left(\frac{\partial u(x, \tau)}{\partial x}\right)\right|^{2} d x \leq\right. \\
\frac{1}{32} \int_{0}^{1}\left|\frac{\partial^{2}}{\partial x^{2}}\left(x\left(\frac{\partial \varphi}{\partial x}\right)\right)\right|^{2} d x+\frac{1}{2} \int_{0}^{\tau} \int_{0}^{1} x^{2} \exp (-c t)\left|\frac{\partial u}{\partial t}\right|^{2} d x d t \\
+\frac{1}{2} \int_{0}^{\tau} \int_{0}^{1} x^{3}|\mathcal{L} u|^{2} d x d t+\frac{1}{2} \int_{0}^{\tau} \int_{0}^{1} \exp (-c t)\left|\frac{\partial^{2} u}{\partial x \partial t}\right|^{2} d x d t
\end{gathered}
$$


Now, combining inequalities (2.9), (2.21), and (2.23), and lemmas, we get

$$
\begin{gathered}
\frac{2}{3} \int_{0}^{\tau} \int_{0}^{1} x^{3}|\mathcal{L} u|^{2} d x d t+\frac{1}{4} \int_{0}^{1} x^{2}|\varphi|^{2} d x+\frac{1}{2} \int_{0}^{1} x\left|\frac{\partial \varphi}{\partial x}\right|^{2} d x \\
+\frac{1}{32} \int_{0}^{1}\left|\frac{\partial^{2}}{\partial x^{2}}\left(x\left(\frac{\partial \varphi}{\partial x}\right)\right)\right|^{2} d x \geq \\
\exp (-c T)\left(\frac{1}{2} \int_{0}^{\tau} \int_{0}^{1} x^{2}\left|\frac{\partial u}{\partial t}\right|^{2} d x d t\right. \\
+\frac{1}{2} \int_{0}^{\tau} \int_{0}^{1}\left|\frac{\partial^{2} u}{\partial x \partial t}\right|^{2} d x d t+\frac{(c-17)}{32} \int_{0}^{\tau} \int_{0}^{1}\left|\frac{\partial^{2}}{\partial x^{2}}\left(x\left(\frac{\partial u}{\partial x}\right)\right)\right|^{2} d x d t \\
+\frac{1}{4} \int_{0}^{\tau} \int_{0}^{1}\left|\frac{\partial^{3}}{\partial x^{3}}\left(x\left(\frac{\partial^{2} u}{\partial x \partial t}\right)\right)\right|^{2} d x d t+\frac{1}{4} \int_{0}^{1} x^{2}|u(x, \tau)|^{2} d x \\
+\frac{1}{2} \int_{0}^{1} x\left|\frac{\partial u(x, \tau)}{\partial x}\right|^{2} d x+\frac{1}{32} \int_{0}^{1} \mid \frac{\partial^{2}}{\partial x^{2}}\left(\left.x\left(\frac{\partial u(x, \tau)}{\partial x}\right)\right|^{2} d x\right. \\
\left.+\frac{c}{2} \int_{0}^{\tau} \int_{0}^{1} x\left|\frac{\partial u}{\partial x}\right|^{2}\right)
\end{gathered}
$$

As the left-hand side of (2.24) is independent of $\tau$, by replacing the right-hand side by its upper bound with respect to in the interval $[0, T]$, we obtain the desired inequality.

\section{Solvability of the problem.}

From estimates (2.1) and (2.5) it follows that the operator $L: E \longrightarrow F$ is continuous and its range is closed in $F$. Therefore, the inverse operator $L^{-1}$ exists and is continuous from the closed subspace $R(L)$ onto $E$, which means that $L$ is a homomorphism from $E$ onto $R(L)$. To obtain the uniqueness of solution, it remains to show that $R(L)=F$. The proof is based on the following lemma.

Lemma 3.1 Let $D_{0}(L)=\left\{u \in D(L) / l u=0, \frac{\partial u(x, T)}{\partial x}=0\right\}$. If for $u \in D_{0}(L)$ and some $\omega$ such that $\omega \in L_{2}(\Omega)$, we have

$\int_{\Omega} x^{4}(1-x) \mathcal{L} u \bar{\omega} d x d t=0$

then $\omega=0$.

Proof. The equality (3.1) can be written as follows

$$
\int_{\Omega} x^{4}(1-x) \frac{\partial u}{\partial t} \bar{\omega} d x d t=\int_{\Omega} x(1-x)\left(\frac{\partial^{3}}{\partial x^{3}}\left(x\left(\frac{\partial^{2} u}{\partial x \partial t}\right)\right)+\frac{\partial^{2}}{\partial x^{2}}\left(x\left(\frac{\partial u}{\partial x}\right)\right)-\frac{\partial^{2} u}{\partial x \partial t}\right) \bar{\omega} d x d t
$$

For $\omega(x, t)$ given, we introduce the function

$v(x, t)=(x-1) \omega(x, t)-x \frac{\partial}{\partial x}((1-x) \omega(x, t))$

Integrating by parts with respect to $\xi$, we obtain

$$
\int_{x}^{1} v(\xi, t) d \xi=\int_{x}^{1}(\xi-1) \omega(\xi, t) d \xi-\int_{x}^{1} \xi \frac{\partial}{\partial \xi}((1-\xi) \omega(\xi, t)) d \xi
$$

which implies that

$\int_{x}^{1} v(\xi, t) d \xi=x(1-x) \omega(x, t)$

Then we have

$$
x^{3} \mathcal{J}_{v}=x^{4}(1-x) \omega(x, t)=N v
$$


which implies that

$\int_{0}^{1} v(x, t) d x=0$

Then from equality (3.2) we have

$$
\begin{aligned}
-\int_{\Omega} \frac{\partial u}{\partial t} N \bar{v} d x d t= & -\int_{\Omega}\left(\frac{\partial^{3}}{\partial x^{3}}\left(x\left(\frac{\partial^{2} u}{\partial x \partial t}\right)\right)+\frac{\partial^{2}}{\partial x^{2}}\left(x\left(\frac{\partial u}{\partial x}\right)\right)\right. \\
& \left.-\frac{\partial^{2} u}{\partial x \partial t}\right)\left(\mathcal{J}_{\bar{v}}\right) d x d t
\end{aligned}
$$

Integrating by parts the second member of the right hand side of (3.5), we get

$$
-\int_{\Omega} \frac{\partial u}{\partial t} N \bar{v} d x d t=\int_{\Omega} A u \bar{v} d x d t
$$

Where

$A u=-\left(\frac{\partial^{2}}{\partial x^{2}}\left(x \frac{\partial^{2} u}{\partial x \partial t}\right)+\frac{\partial}{\partial x}\left(x \frac{\partial u}{\partial x}\right)-\frac{\partial u}{\partial t}\right)$

When we introduce the smoothing operators $J_{\varepsilon}^{-1}$ and $\left(J_{\varepsilon}^{-1}\right)^{*}$, with respect to [13] then these operators provide the solution of the problems

$$
\begin{gathered}
\varepsilon \frac{d g_{\varepsilon}(t)}{d t}+g_{\varepsilon}(t)=g(t) \\
\left.g(t)_{\varepsilon}\right|_{t=0}=0
\end{gathered}
$$

And

$$
\begin{gathered}
-\varepsilon \frac{d g_{\varepsilon}^{*}(t)}{d t}+g_{\varepsilon}^{*}(t)=g(t) \\
\left.g_{\varepsilon}^{*}(t)\right|_{t=T}=0
\end{gathered}
$$

The solution have the following properties: for $g \in L_{2}(0, T)$, the functions $g_{\varepsilon}=\left(J_{\varepsilon}^{-1}\right) g$ and $g_{\varepsilon}^{*}=\left(J_{\varepsilon}^{-1}\right)^{*} g$ are in $W_{2}^{1}(0, T)$ such that $\left.g(t)_{\varepsilon}\right|_{t=0}=0$ and $\left.g^{*}(t)_{\varepsilon}\right|_{t=T}=0$. Moreover, $J_{\varepsilon}^{-1}$ commutes with $\frac{\partial}{\partial t}$, so $\int_{0}^{T}\left|g_{\varepsilon}-g\right|^{2} \longrightarrow 0$ and $\int_{0}^{T}\left|g_{\varepsilon}^{*}-g\right|^{2} \longrightarrow 0$, for $\varepsilon \longrightarrow 0$.

Replacing in (3.6) $u$ by the smoothed function $\left(J_{\varepsilon}^{-1}\right) u$, using the relation

$A(t) J_{\varepsilon}^{-1}=J_{\varepsilon}^{-1} A(\tau)+\varepsilon J_{\varepsilon}^{-1} \frac{\partial A}{\partial \tau} J_{\varepsilon}^{-1}$

And using properties of the smoothing operators we get

$$
-\int_{\Omega} u\left(\overline{N \frac{\partial v_{\varepsilon}^{*}}{\partial t}}\right) d x d t=\int_{\Omega} A u \overline{v_{\varepsilon}^{*}} d x d t+\varepsilon \int_{\Omega} \frac{\partial A}{\partial t} u_{\varepsilon} \overline{v_{\varepsilon}^{*}} d x d t .
$$

Passing to the limit, (3.7) is satisfied for all functions satisfying the conditions (1.3)-(1.4) such that

$$
\frac{\partial^{i}}{\partial x^{i}}\left(\frac{\partial u}{\partial x}\right) \in L_{2}(\Omega), \frac{\partial u}{\partial x} \in L_{2}(\Omega), \frac{\partial^{i}}{\partial x^{i}}\left(\frac{\partial^{2} u}{\partial x \partial t}\right) \in L_{2}(\Omega) \quad \text { for } \quad 0 \leq i \leq 3
$$

The left-hand side of (3.9) is a continuous linear functional of $u$. Hence the function $v_{\varepsilon}^{*}$ has the derivative

$\frac{\partial v_{\varepsilon}^{*}}{\partial x} \in L_{2}(\Omega), \frac{\partial^{i}}{\partial x^{i}}\left(\frac{\partial v_{\varepsilon}^{*}}{\partial x}\right) \in L_{2}(\Omega) \quad$ for $\quad 0 \leq i \leq 3$

and the following condition is satisfied

$$
\left.\frac{\partial^{i} v_{\varepsilon}^{*}}{\partial x^{i}}\right|_{x=1}=\left.\frac{\partial^{i} v_{\varepsilon}^{*}}{\partial x^{i}}\right|_{x=0}=0, i=\overline{0,2}
$$


In addition $v^{*}$ satisfies the integral condition (1.4). Putting $u=\int_{0}^{t} v_{\varepsilon}^{*}(x, \tau) d \tau$ in (3.6) and using (3.8), we obtain

$$
\begin{gathered}
-\int_{\Omega} v_{\varepsilon}^{*} \overline{N v} d x d t=\int_{\Omega} A u \overline{v_{\varepsilon}^{*}} d x d t-\varepsilon \int_{\Omega} A u \frac{\partial \overline{v_{\varepsilon}^{*}}}{\partial t} d x d t \\
=\int_{\Omega} A u \frac{\partial \bar{u}}{\partial t} d x d t-\varepsilon \int_{\Omega} A u \frac{\partial \overline{v_{\varepsilon}^{*}}}{\partial t} d x d t
\end{gathered}
$$

Integrating by parts each term in the right-hand side of (3.11), we have

$$
\begin{aligned}
& \operatorname{Re} \int_{\Omega} A u \frac{\partial \bar{u}}{\partial t} d x d t=\int_{\Omega}\left|v_{\varepsilon}^{*}\right|^{2} d x d t \geq 0 \\
& \operatorname{Re}\left(-\varepsilon \int_{\Omega} A u \frac{\partial \overline{v_{\varepsilon}^{*}}}{\partial t} d x d t\right)=\varepsilon \int_{\Omega} x\left|\frac{\partial v_{\varepsilon}^{*}}{\partial x}\right|^{2} d x d t+\frac{\varepsilon}{2} \int_{0}^{1}\left|v_{*}^{\varepsilon}\right|^{2} d x \geq 0
\end{aligned}
$$

Now, using (3.12) and (3.13) in (3.11) we have

$\operatorname{Re} \int_{\Omega} v_{\varepsilon}^{*} \overline{N v} d x d t \leq 0$

then

$\operatorname{Re} \int_{\Omega} v \overline{N v} d x d t \leq 0$ as $\varepsilon$ approaches zero.

Since $\int_{\Omega} x^{3}|v|^{2} d x d t=0$, we conclude that $v=0$, hence $\omega=0$, what finishes the proof of the lemma. The range $R(L)$ of the operator $L$ coincides with $F$.

Proof. Since $F$ is a Hilbert space, we have $R(L)=F$ if and only if the following implication is satisfied:

$$
\begin{gathered}
\int_{\Omega} x^{4} \mathcal{L} u \bar{f} d x d t+\int_{0}^{1}\left(\frac{\partial^{2}}{\partial x^{2}}\left(x\left(\frac{\partial l u}{\partial x}\right)\right) \frac{\partial^{2}}{\partial x^{2}}\left(x\left(\frac{\partial \bar{\varphi}}{\partial x}\right)\right)\right. \\
+x\left(\frac{\partial l u}{\partial x}\right)\left(\frac{\partial \bar{\varphi}}{\partial x}\right)+x^{2}(l u)(\bar{\varphi}) d x=0
\end{gathered}
$$

for arbitrary $u \in E$ and $\mathcal{F}=(f, \varphi) \in F$, implies that $f$ and $\varphi$ are zero. Putting $u \in D\left(L_{0}\right)$ in (3.15), Taking $\omega=\frac{f}{(1-x)}$, and using lemma 3.1 we obtain that $\frac{f}{(1-x)}=0$, then $f=0$. Consequently, $\forall u \in D(L)$ we have

$\int_{\Omega} x^{4} \mathcal{L} u \bar{f} d x d t=0$

The range of the trace operator $l$ is everywhere dense in a Hilbert space with norm

$\left[\int_{0}^{1}\left|\frac{\partial^{2}}{\partial x^{2}}\left(x\left(\frac{\partial \varphi}{\partial x}\right)\right)\right|^{2}+x\left|\left(\frac{\partial \varphi}{\partial x}\right)\right|^{2}+x^{2}|\varphi|^{2} d x\right]^{\frac{1}{2}}$.

therefore $\varphi=0$, and the present proof is completed.

\section{References}

[1] A.M.Al-kadhi, A Class of Hyperbolic Equations with Nonlocal Conditions, Int Journal of Math Analysis, 10 (2008) 491 - 498.

[2] A.Bouziani, Mixed problem with integral boundary condition for a certain parabolic equations , J Applied Mathematics and Stochastic Analysis, 3 (1996) 323-330.

[3] A.Bouziani, N.E.Benouar, problème mixte avec conditions intégrales pour une classe d'équations hyperboliques, Bull Belg Math Soc, 3 (1996) 137-145.

[4] L.Bougoffa, A third-order non-local problem with non local conditions, IJMMS ,28 (2004) 1503-1507 
[5] M.Bouzit, N.Teyar, A Class of Third Order Parabolic Equations with Integral Conditions, Int Journal of Math Analysis, 18 (2009) 871 - 877.

[6] M.Denche, On non-local problems for ordinary differential equations and on a non local parabolic transmission problem , Journal of Applied Mathematics and Stochastic Analysis, 3 (1999) 279-291 .

[7] M.Denche, A.L.Marhoune, Mixed problem with integral boundary condition for a high order mixed type partial differential equation, J Appl Math Stochastic Anal, 1 (2003) 69-79.

[8] M.Denche, A.L.Marhoune, Mixed problem with non local boundary conditions for a third-order . partial differential equation of mixed type, Int J Math Sci, 7 (2001) 417-426.

[9] M.Denche, A.L.Marhoune, High-order mixed-type differential equations with weighted integral boundary conditions, Electronic Journal of Differential Equations, 60 (2000) 1-10.

[10] M.Denche, A.Memou, Boundary value problem with integral conditions for a linear third-order equation, Journal of Applied Mathematics, 11 (2003) 553 - 567.

[11] S.Mesloub, A.Bouziani, On a class of singular hyperbolic equation with a weighted Iintegral condition, Int $J$ mathE math Sci, 3(1999) 511-519.

[12] S.Mesloub, A.Bouziani, N.Kechkar, A strong solution of an evolution problem with inyegral conditions, Georgian Mathematical Journal, 1 (2002) 149 -159.

[13] N.I.Yurchuk, Mixed problem with an integral condition for certain parabolic equations, Diff Equ, 22 (1986) $1457-1463$. 